\title{
CORRELATION BETWEEN ERYTHROCYTE SUPEROXIDE DISMUTASE AND CATALASE LEVELS AND PERIPHERAL NERVE CONDUCTION IN DIABETIC NEUROPATHY PATIENTS
}

\author{
Gordana Đorđević1,2, Srđan Sretenović3, Jelena Stamenović1,2, \\ Aleksandar Stojanov ${ }^{1}$
}

Neurology Clinic, Clinical Center Niš, Serbia ${ }^{1}$

Universityof Niš, Faculty of Medicine, Niš, Serbia ${ }^{2}$

Medihelp Clinical Neurology private practice, Belgrade, Serbia ${ }^{3}$

Contact: Gordana Đorđević

Nikole Kopernika 28,18000 Niš

e-mail: gordanadjor@yahoo.com

Reduced systemic antioxidant defence is considered to play an important mediating role in the pathogenesis of diabetic neuropathy.

The aim of this study was to determine superoxide dismutase (SOD) and catalase (CAT) levels in the erythrocytes of patients with type 2 diabetes mellitus (DM) and diabetic distal symmetrical polyneuropathy (DDSP), and to analyze the connection between the activity of these antioxidative enzymes and peripheral nerve function.

This study involved 100 patients with type 2 DM and signs of DDSP, as well as the control group of 50 healthy subjects and 40 diabetic patients without DDSP. The evaluation of DDSP was based on physical examination and nerve conduction studies. The degree of peripheral nerve dysfunction was estimated by analyzing and scoring sensory and motor nerve conduction parameters.

Laboratory analyses involved erythrocyte SOD and CAT values. SOD values were significantly lower in the patients in comparison with the control group $(p<0.0001)$ and diabetic patients without DDSP. The values of erythrocyte CAT were also reduced in diabetic neuropathy patients compared with the controls and patients without DN, although the reduction was not statistically significant. A number of electroneurographic parameters correlated significantly with SOD and CAT levels in the studied patients. Erythrocyte SOD and CAT values were reduced in patients with type 2 DM and DDSP and they correlated with certain electroneurographic parameters of peripheral nerve conduction, which suggested that oxidative stress was potentially implicated in the development of diabetic neuropathy. Acta Medica Medianae 2017;56(2):78-84.

Key words: diabetes mellitus, diabetic neuropathy, oxidative stress, superoxide dismutase, catalase. 